\title{
Dihydroartemisinin attenuates autoimmune thyroiditis by inhibiting the CXCR3/PI3K/AKT/NF-KB signaling pathway
}

\author{
Huijuan Liu ${ }^{1,2, *}$, Qin Tian ${ }^{1, *}$, Xiaoyu Ai ${ }^{1, *}$, Yuan Qin ${ }^{1}$, Zhanhong Cui ${ }^{1}$, Meng $\mathbf{L i}^{1}$, \\ Jiahuan Yang ${ }^{1}$, Denghui Zhai ${ }^{1}$, Yanrong Liu ${ }^{3}$, Shuang Chen ${ }^{3}$, Jing Meng ${ }^{1}$, Tao Sun ${ }^{1,3}$, \\ Honggang Zhou ${ }^{1,3}$ and Cheng Yang ${ }^{1,3}$ \\ ${ }^{1}$ State Key Laboratory of Medicinal Chemical Biology and College of Pharmacy, College of Life Sciences, Nankai University, \\ Tianjin, China \\ ${ }^{2}$ College of Life Sciences, Nankai University, Tianjin, China \\ ${ }^{3}$ Tianjin Key Laboratory of Molecular Drug Research, Tianjin International Joint Academy of Biomedicine, Tianjin, China \\ *These authors have contributed equally to this work
}

Correspondence to: Cheng Yang, email: cyang66_2001@yahoo.com

Honggang Zhou, email: honggang.zhou@vip.126.com

Tao Sun, email: sunrockmia@hotmial.com

Keywords: DHA; AIT; CXCR3; PI3K; NF-KB

Received: September 19, $2017 \quad$ Accepted: November 13, $2017 \quad$ Published: December 01, 2017

Copyright: Liu et al. This is an open-access article distributed under the terms of the Creative Commons Attribution License 3.0 (CC BY 3.0), which permits unrestricted use, distribution, and reproduction in any medium, provided the original author and source are credited.

\section{ABSTRACT}

Dihydroartemisinin (DHA) is the first generation of naturally occurring artemisinin derivatives with antimalarial activity. Recent research showed that this drug also features immunosuppressive and anti-inflammatory properties. Autoimmune thyroiditis (AIT) is a common organ-specific autoimmune disease with no available effective drug treatment. In this study, we investigated effects of DHA on AIT in vitro and in vivo. Results showed that DHA can visibly reduce antithyroglobulin antibody and thyroid peroxidase antibody levels and regulate $T$ helper cells (Th) 1/Th2 imbalance of experimental AIT mice. DHA also dose-dependently suppressed proliferation of lymphocytes induced by lipopolysaccharide and concanavalin A. DHA inhibited binding of C-X-C chemokine ligand 10 (CXCL10) and its receptor (C-X-C motif) receptor 3 (CXCR3), thus inhibiting calcium flow. DHA can also reduce expression levels of PI3-kinase (PI3K), p-PI3K, protein kinase B (AKT), p-AKT, nuclear factor (NF)-KB/ p65, and $p-N F-k B / p 65$. In conclusion, DHA may serve as treatment drug for AIT by inhibiting the CXCR3/PI3K/AKT/NF-kB signaling pathway.

\section{INTRODUCTION}

Autoimmune thyroiditis (AIT) is a common organspecific autoimmune disease and a type of autoimmune thyroid disease (AITD). Clinical types of AITD include Hashimoto's thyroiditis (HT), Grave's disease, and atrophic thyroiditis; many factors, such as genetic and environmental factors, are suspected to play important roles in AITD [1]. HT, which is chronic lymphocytic thyroiditis, is the most common clinical type with high prevalence of $13.4 \%$. Prevalence of HT is high in middleaged women, especially those in menopausal stage [2].
The most common manifestations of AIT comprise extensive lymphocyte infiltration, follicular collapse, and follicular cell degeneration [3]. Clinical features are characterized by enlargement of the thyroid gland with serum antithyroglobulin antibody (TGAb) and thyroid peroxidase antibody (TPOAb), of which TPOAb bears more importance [4]. Irregular treatment of patients will lead to increased thyroid autoantibodies, serious autoimmune inflammation damage to structure and function of thyroid follicular cells, and development of a series of diseases, such as hyperthyroidism, hypothyroidism, thyroid nodules, and thyroid cancer 
(TC) [5]. A significant association exists between HT and TC; statistics shows that incidence of TC reaches $45.7 \%$ in patients with HT and $29 \%$ in those without [6]. At present, primary treatment methods for AITD include thyroid hormone replacement therapy (only for patients with hypothyroidism), immunosuppressive therapy, and glucocorticoid therapy. These treatments pose different side effects. Therefore, novel and low-toxicity drugs should be developed for AITD treatment.

CXC chemokine ligand 10 (CXCL10) is an interferon (IFN)- $\gamma$-inducible chemokine that belongs to the ELR-CXC subfamily chemokine. CXCL10 exerts its function through binding to chemokine $(\mathrm{C}-\mathrm{X}-\mathrm{C}$ motif) receptor 3 (CXCR3), which is a seven-trans-membrane receptor coupled to $\mathrm{G}$ proteins. CXCL10 and CXCR3 play important roles in processes of AITD. Therefore, blocking CXCR3 may abrogate the related inflammatory process. CXCL10/CXCR3 may be considered a novel therapeutic target of thyroiditis [7-9].

Dihydroartemisinin (DHA) is the first generation of artemisinin derivatives with antimalarial activity that is 10 times higher than artemisinin. Studies showed that DHA exhibits anti-inflammatory and immunosuppressive effects with better efficiency and lower toxicity than artemisinin. Results of Youyou Tu's group showed good curative effect of DHA treatment on systemic lupus erythematosus (SLE) [10]. And its clinical trials for SLE has been approved by SFDA in 2016. In this article, we investigate therapeutic effects of DHA on AIT and discuss its effect on CXCL10/ CXCR3 signaling pathway.

\section{RESULTS}

\section{DHA improves overall health of experimental AIT (EAT) mice and possesses immunity adjustment function}

Figure 1A shows modeling and administration methods used in EAT mice. Weight of mice in all groups was monitored every other day until they were sacrificed. A significant difference was observed between body weight of the model and blank groups (Figure 1B). Body weight of mice in the model group decreased dramatically. A significant difference in body weight was detected between DHA treatment group and model group. DHA significantly improved body weight and survival state of EAT mice, whereas EAT mice in the Dex group did not present significant improvement in weight.

Thymus and spleen index of all DHA treatment groups markedly decreased compared with those of the model group (Figures 1C, 1D). By contrast, the positive drug dexamethasone can only reduce thymus coefficient. Results showed that TGAb and TPOAb levels in serum of the model group were significantly higher than those in the blank group and were remarkably reduced in DHA treatment groups (Figures 1E, 1F).

\section{DHA inhibits infiltration of inflammatory cells in the thyroid gland of EAT mice}

Various degrees of mononuclear cell infiltration were observed in EAT mice by hematoxylin and eosin (H\&E) staining (Figures $2 \mathrm{~A}-2 \mathrm{~F}$ ), with scores ranging from 0 to 4 (Figure 2G). Figure 2A shows a normal thyroid tissue without infiltration of inflammatory cells and thyroid follicle destruction. Thyroid follicle destruction caused by infiltration of inflammatory cells can be observed in most thyroid tissues in the model group (Figure 2B). Both DHA and Dex treatments can alleviate symptoms of inflammatory cell infiltration, with high dose and medium dose of DHA presenting a particular significance (Figures 2C-2F). Mean scores for mononuclear cell infiltration significantly reduced in DHA treatment groups (Figure 2G). This result indicates that DHA can reduce infiltration of intrathyroid mononuclear cells.

\section{DHA regulates $\mathrm{Th} 1 / \mathrm{Th} 2$ cytokines}

Th1 type (IFN- $\gamma$ and interleukin (IL)-2) and Th2 type (IL-4 and IL-6) cytokines were determined by enzyme-linked immunosorbent assay (ELISA). Contents of IFN- $\gamma$ and IL-2 cytokines (Figure 3A, 3B) in sera of the model group increased significantly, whereas IL-4 and IL-6 cytokines decreased remarkably compared with that of the blank group (Figure 3C, 3D). DHA-treated group and Dex group showed significantly reduced contents of IFN- $\gamma$ and IL-2 in serum of EAT mice (Figures 3A, $3 \mathrm{~B}$ ), whereas DHA $\mathrm{H}$ group exhibited significantly increased IL-4 and IL-6 contents compared with the model group (Figure 3C, 3D). This phenomenon indicates the imbalance between Th1 and Th2 in EAT mice, and this condition was alleviated by DHA treatment. The mRNA expressions of $\mathrm{T}$ subset-specific transcription factors and typical cytokines in the spleen were determined. As shown in Figures 3E-3F, relative mRNA expressions of Th1 typical cytokine IFN- $\gamma$ and the transcription factor T-box transcription factor 21(T-bet) were significantly inhibited in DHA and Dex groups. High doses of DHA upregulated relative mRNA expression of IL-4 in the spleen of EAT mice (Figure $3 \mathrm{G}$ ), and relative mRNA expression level of GATA-binding protein 3 (GATA3) was also significantly higher in the DHA-treated group (Figure 3H).

\section{DHA suppresses lymphocyte proliferation and chemotactic movement}

DHA dose-dependently decreased proliferation of lymphocytes activated by lipopolysaccharide (LPS) and concanavalin A (Con A) (Figure 4A). Chemotactic movements of lymphocytes were compared by measuring their transmigration through transwell assay in response to different concentrations of DHA. Results showed that DHA significantly inhibited chemotaxis of lymphocytes (Figure 4B). 
DHA interrupts binding of chemokine CXCL10 to CXCR3 and inhibits the PI3-kinase (PI3K)/ protein kinase B (AKT)/nuclear factor (NF)-kB pathway

The combination of chemokine CXCL10 and chemokine receptor CXCR3 can cause changes in intracellular calcium concentration. Results showed that DHA visibly decreased levels of calcium flow intensity and restrained binding of CXCL10 and CXCR3 $(\mathrm{EC} 50=3.763 \mu \mathrm{M})$ (Figures $4 \mathrm{C}, 4 \mathrm{D})$. Transcriptional activation activity of NF-kB/p65 in HEK293 cells was inhibited by DHA according to results of dual-luciferase assay (Figure 4E). Phorbol-12-myristate-13-acetate (PMA)
A

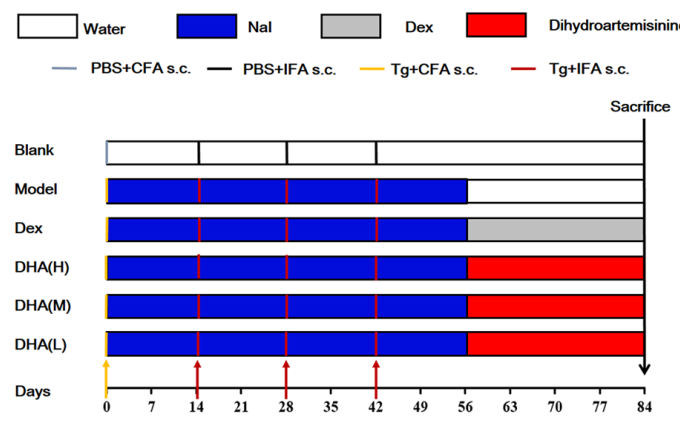

C

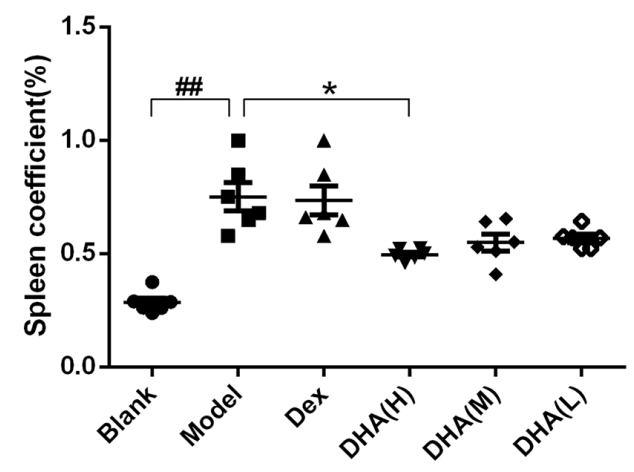

E

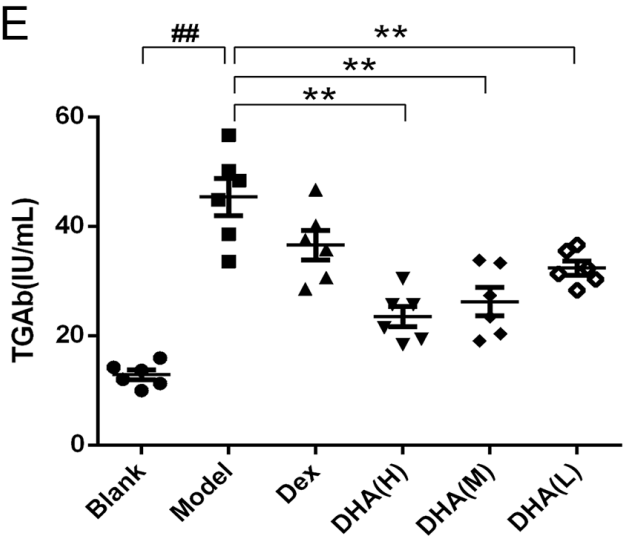

B

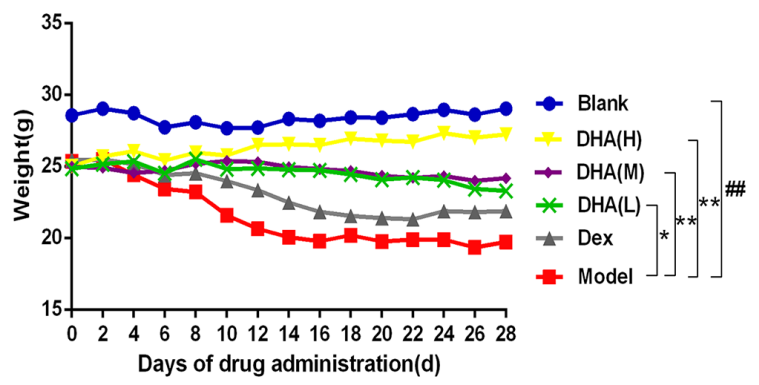

D

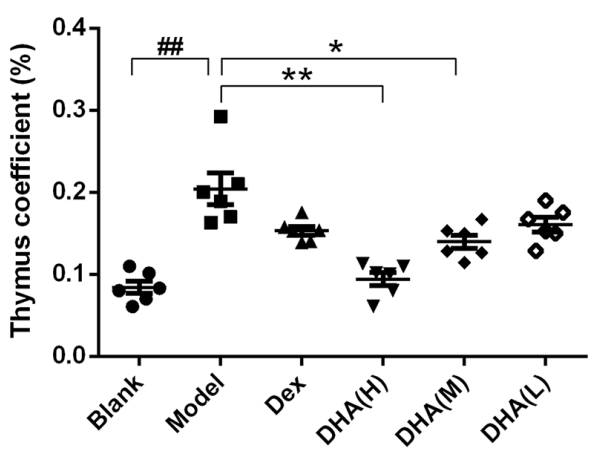

$\mathrm{F}$

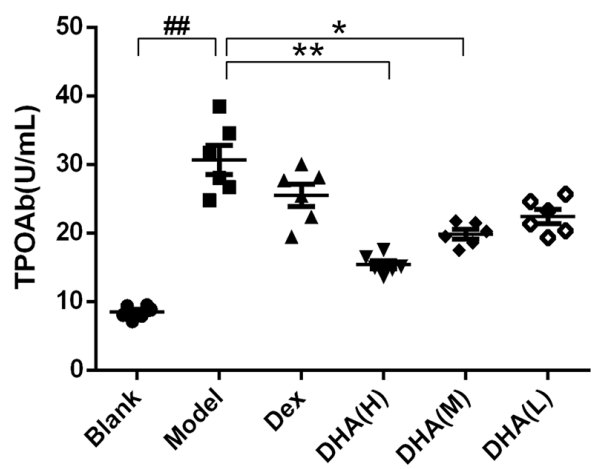

Figure 1: Effect of DHA on a C57BL/6J EAT mouse model. (A) Workflow of animal experiment. (B) Body weights (g) of EAT mice with DHA administration. (C and D) Effects of DHA on spleen and thymus coefficients of EAT mice. (E and F) TGAb and TPOAb contents in sera of EAT mice after DHA treatment. Values are presented as mean $\pm \mathrm{SD}$. ${ }^{\#} \mathrm{P}<0.01$ versus control group. ${ }^{*} \mathrm{P}<0.05,{ }^{* *} \mathrm{P}<$ 0.01 versus model group. 
promoted protein expression of lymphocytes according to Western blot. Compared with the group induced by PMA, expression of CXCR3 downstream proteins, such as PI3K, p-PI3K, AKT, p-AKT, NF-kB/p65, and p-NF-kB/p65, were significantly reduced by DHA (Figures $4 \mathrm{~F}, 4 \mathrm{G}$ ).

\section{DHA regulates immune and inflammatory signaling pathways}

As observed from the Kyoto Encyclopedia of Genes and Genomes (KEGG) analysis (Figure 5A), DHA affects
A

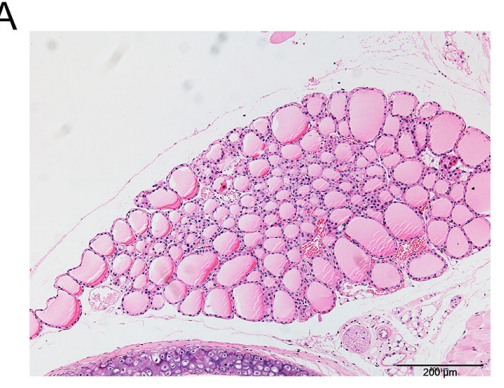

C

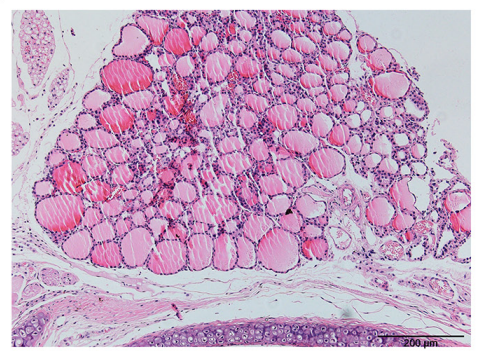

Dex

E

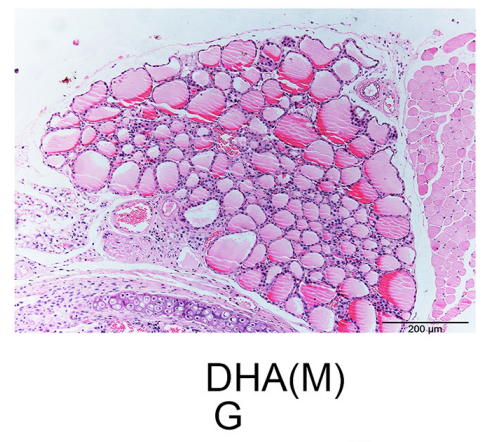

B

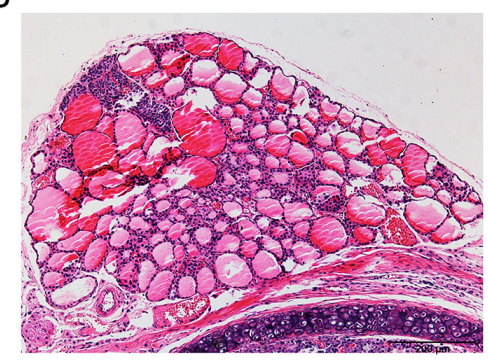

Model

D

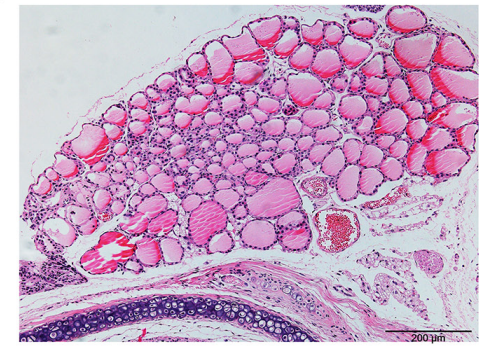

$\mathrm{DHA}(\mathrm{H})$

$\mathrm{F}$

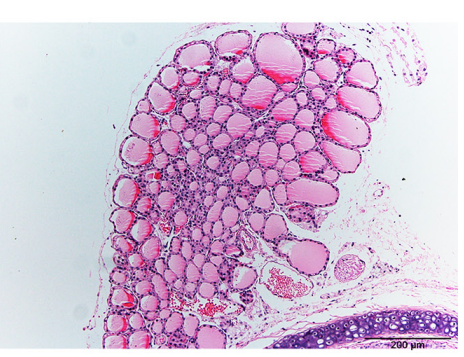

$\mathrm{DHA}(\mathrm{L})$

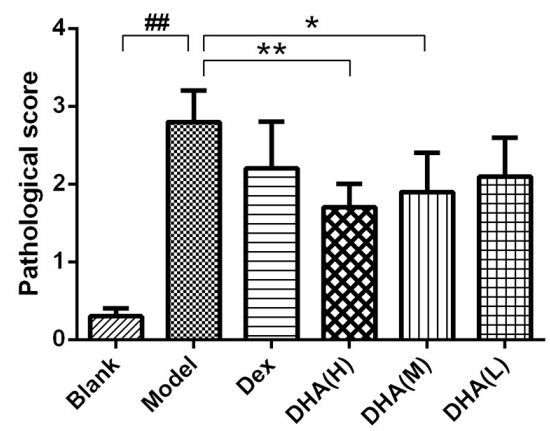

Figure 2: Histopathological changes in the thyroid gland of mice in experimental groups. (A) Thyroid gland architecture of normal control mice (H\&E, 100× magnification). (B) Tg- and NaI-induced EAT model mice showed a large number of inflammatory cell infiltrate $(\mathrm{H} \& \mathrm{E}, 100 \times)$. (C) Treatment with dexamethasone slightly inhibited infiltration of lymphocytes (H\&E, 100×). (D) At $20 \mathrm{mg} /$ $\mathrm{kg} /$ day, DHA significantly inhibited lymphocyte infiltration (H\&E, 100×). (E) At $10 \mathrm{mg} / \mathrm{kg} / \mathrm{day}$, DHA can inhibit lymphocyte infiltration (H\&E, 100×). (F) At $5 \mathrm{mg} / \mathrm{kg} /$ day, DHA slightly inhibited lymphocyte infiltration (H\&E, 100×). (G) Analysis of areas of infiltration of inflammatory cells in experimental groups. Values are presented as mean $\pm \mathrm{SD}$. ${ }^{\# \#} \mathrm{P}<0.01$ versus control group. ${ }^{*} \mathrm{P}<0.05,{ }^{* *} \mathrm{P}<0.01$ versus model group. 
A

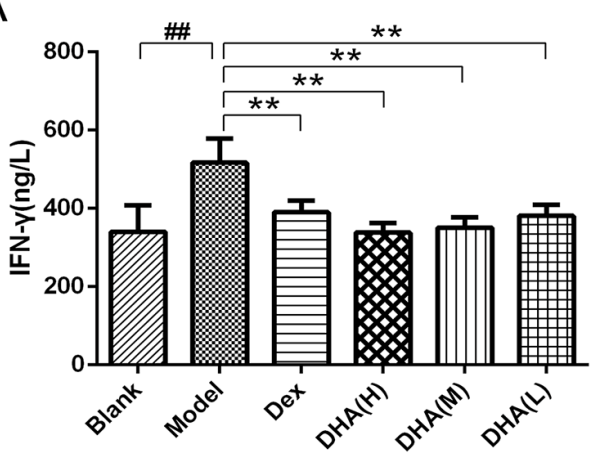

$\mathrm{C}$

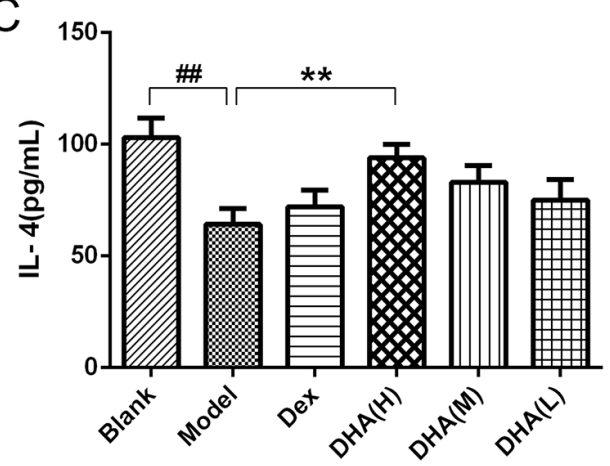

$\mathrm{E}$

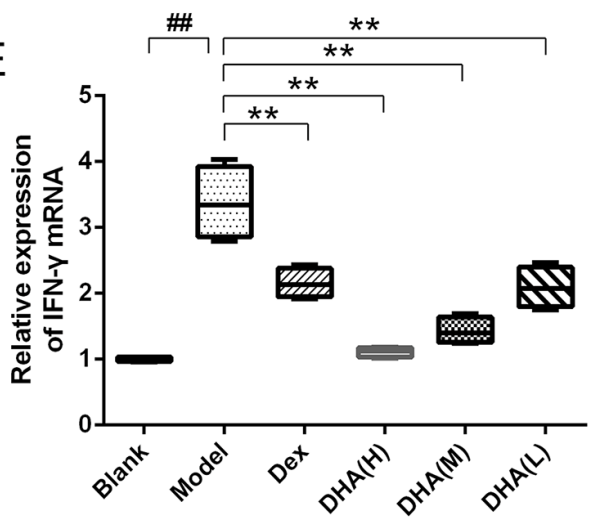

G

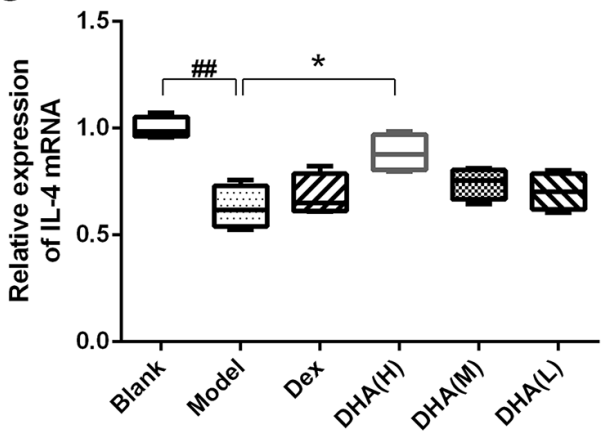

B

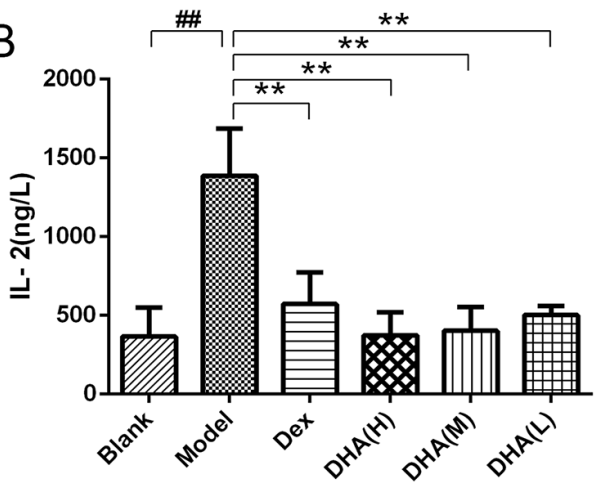

D
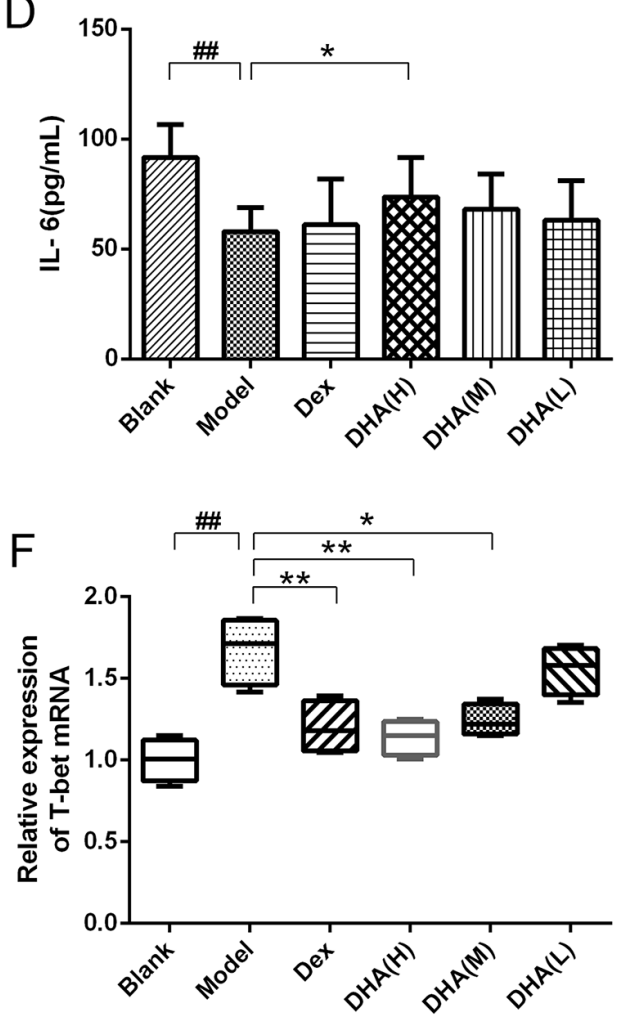

$\mathrm{H}$

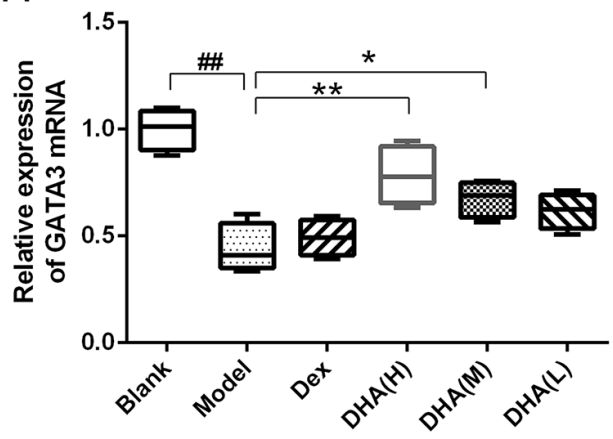

Figure 3: Effect of DHA on Th1/Th2 cytokine balance. (A and B) Contents of Th1 type cytokines IFN- $\gamma$ and IL-2 in sera of EAT mice. (C and D) Contents of Th2 type cytokines IL-4 and IL-6 in sera of EAT mice. (E and F) Relative mRNA expressions of Th1 typical cytokine IFN- $\gamma$ and transcription factor T-bet in experimental groups. (G and H) Relative mRNA expression of Th2 typical cytokine IL-4 and transcription factor GATA3 in experimental groups. Values are presented as mean $\pm \mathrm{SD} .{ }^{\# \mathrm{P}}<0.01$ versus control group. ${ }^{*} \mathrm{P}<0.05,{ }^{* *}$ $\mathrm{P}<0.01$ versus model group. 

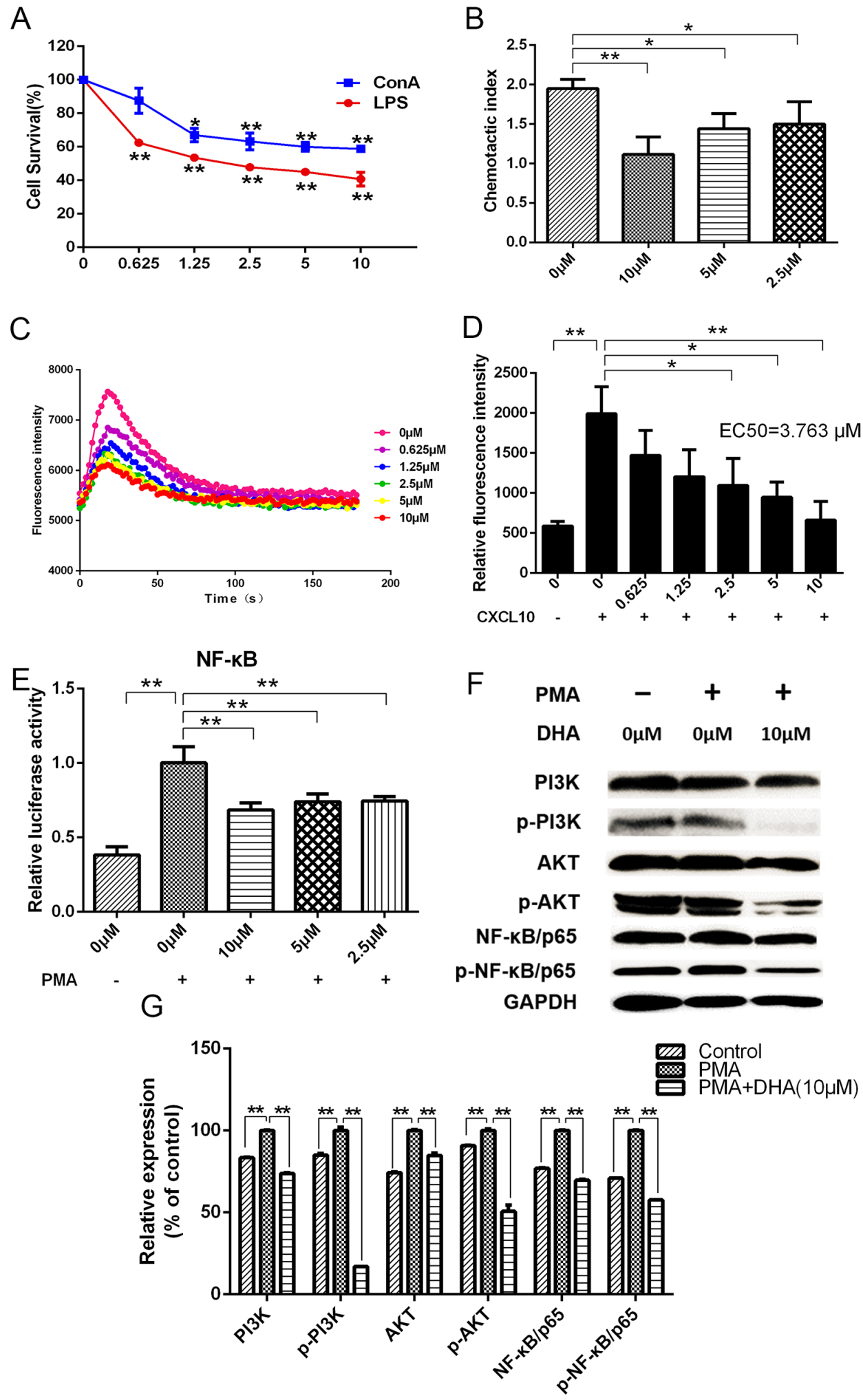

Figure 4: (A) Effects of DHA on lymphocyte proliferation. (B) Effects of DHA on chemotaxis index of lymphocytes. (C and D) Effects of DHA on calcium flow signals during binding of CXCL10 to CXCR3. (E) Dual-luciferase assay results for the effects of DHA on transcriptional activation activity of NF- $\mathrm{KB}$ in lymphocytes induced by PMA. (F and G) Western blot assay results for the effects of DHA on PI3K, p-PI3K, AKT, p-AKT, NF-kB/p65, and p-NF-kB/p65 expressions in lymphocytes induced by PMA. 
A

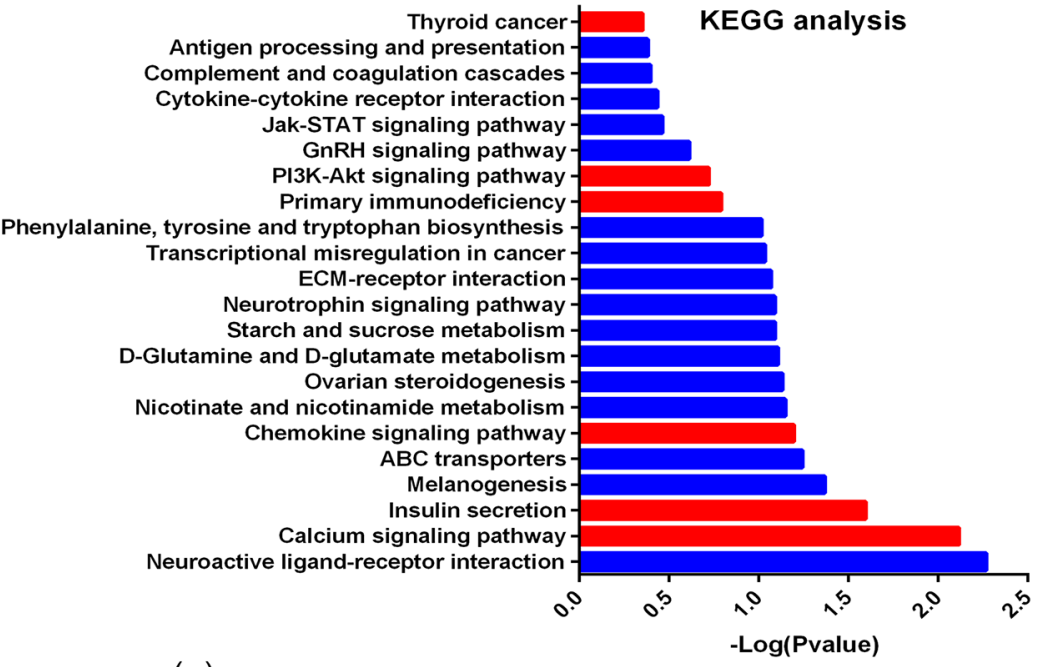

B

(a)
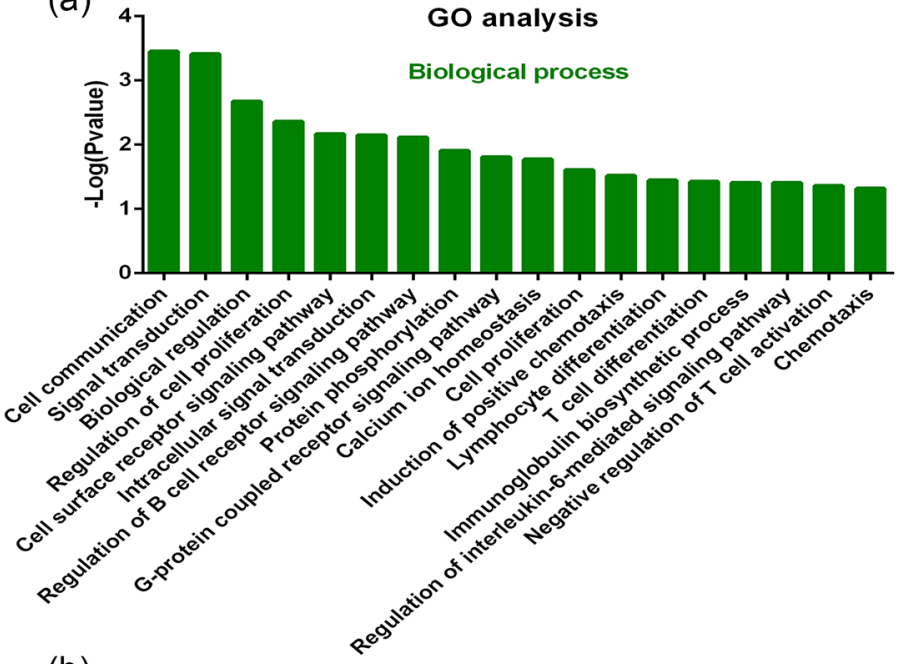

(b)
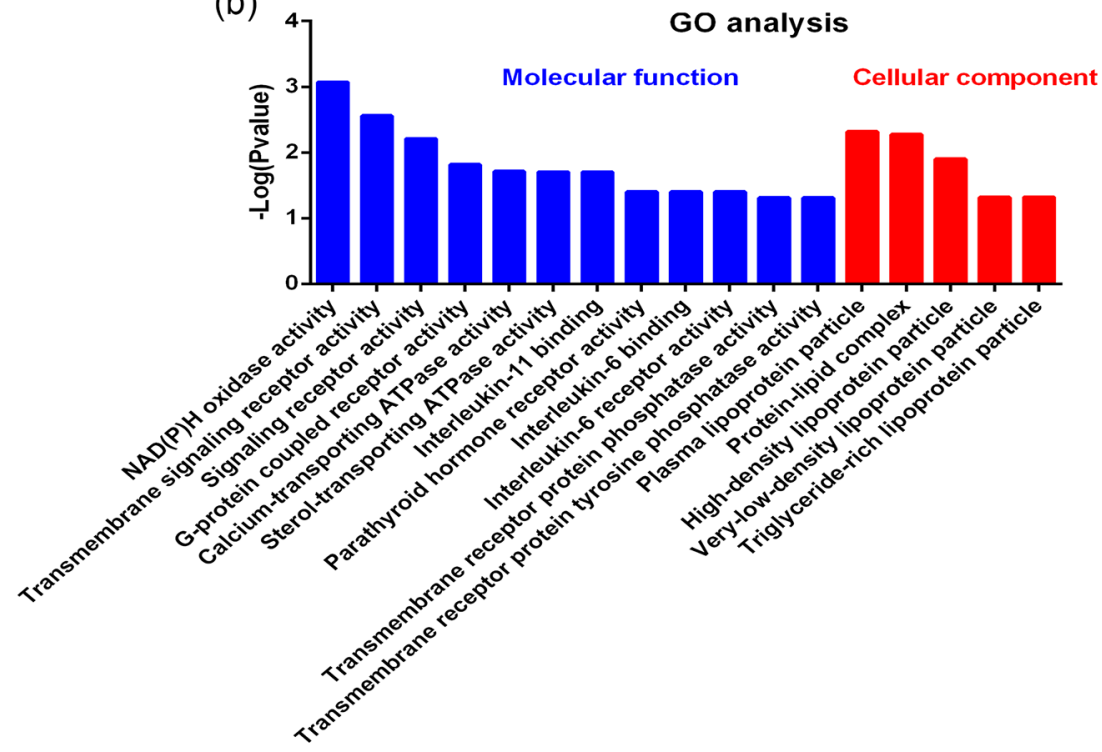

Figure 5: Effects of DHA on lymphocyte expression detected by microarray. (A) KEGG analysis for effects of DHA on signal pathways. (B) GO analysis for the effects of DHA on biological process (a), molecular function, and cellular component (b). 

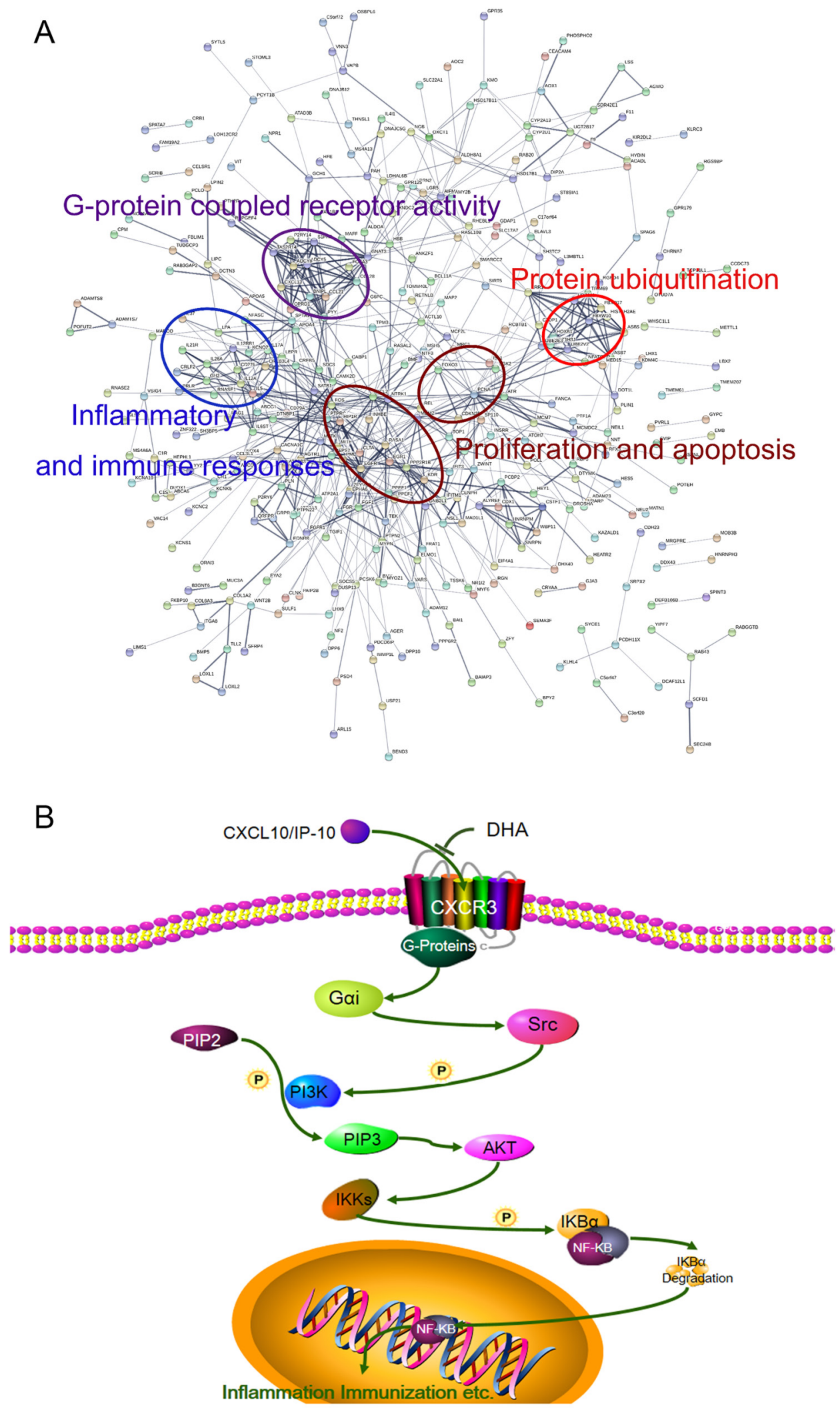

Figure 6: (A) Protein-protein interaction networks of differentially expressed proteins. Significantly changed proteins correlated with $\mathrm{G}$ protein-coupled receptor activity, inflammation and immune responses, proliferation and apoptosis, and protein ubiquitination. (B) DHA signaling pathway in treatment of AIT. 
Table 1: Primer sequences for Q-PCR

\begin{tabular}{|c|c|}
\hline Gene & Primers $\left(5^{\prime}-3^{\prime}\right)$ \\
\hline \multirow[t]{2}{*}{ IFN- $\gamma$} & 5'- CGGCACAGTCATTGAAAGCCTA-3' \\
\hline & 5'- GTTGCTGATGGCCTGATTGTC-3' \\
\hline \multirow[t]{2}{*}{ T-bet } & 5'-GTTCAACCAGCACCAGACAGAG-3' \\
\hline & 5'-TGGTCCACCAAGACCACATC-3' \\
\hline \multirow[t]{2}{*}{ IL-4 } & 5'-ACGGAGATGGATGTGCCAAAC-3' \\
\hline & 5'-AGCACCTTGGAAGCCCTCAGA-3' \\
\hline \multirow[t]{2}{*}{ GATA3 } & 5'-GGATGTAAGTCGAGGCCCAAG-3' \\
\hline & 5'-ATTGCAAAGGTAGTGCCCGGTA-3' \\
\hline \multirow[t]{2}{*}{ GAPDH } & 5'-ACTCCACTCACGGCAAATTC-3' \\
\hline & 5'-TCTCCATGGTGGTGAAGACA-3' \\
\hline
\end{tabular}

a large number of signal pathways, such as calcium signaling pathway, chemokine signaling pathway, primary immunodeficiency, PI3K/AKT signaling pathway, and TC. Gene Ontology (GO) analysis results showed the mainly influenced functions in biological processes, molecular functions, and cellular components. As shown in Figure $5 \mathrm{~A}$, biological processes, such as cell communication, signal transduction, lymphocyte differentiation, and chemotaxis, were notably influenced by DHA. Figure 5B shows molecular function and cellular components, such as G-protein-coupled receptor activity, plasma lipoprotein particle, IL-6 binding, and IL-6 receptor activity, that are affected by DHA. Further analysis revealed that the modified proteins are mainly correlated with $\mathrm{G}$ proteincoupled receptor activity, inflammation and immune responses, proliferation and apoptosis, and protein ubiquitination (Figure 6A).

\section{DISCUSSION}

Incidence of AIT in female is significantly higher than that in male [11]. EAT induced by thyroglobulin (Tg) immunization and $\mathrm{NaI}$ is a classical and widely used animal model in studies of AIT pathogenesis. Using the EAT mouse model, the current study investigated therapeutic effects of DHA on AITD and its potential mechanisms. In this study, overall health of EAT mice improved after administration of DHA, and extent of mononuclear cell infiltration in the thyroid was significantly lower in DHA treatment group in comparison with that of model group. Specifically, blood analysis also showed remarkably lower TGAb and TPOAb levels in the DHA treatment group than in the model group. Weights of spleen and thymus serve as important indices of immune function [12]. Our findings indicated that spleen and thymus coefficients decreased significantly in a dosedependent manner in DHA-treated mice, suggesting that
DHA probably performs immunosuppressive functions. These results indicate that treatment with DHA improved the condition of EAT mice by inhibiting immune and lymphocyte infiltration.

Based on their respective patterns of cytokine production, CD4+ (Th) cells consist of at least two different subsets, namely, Th1 and Th2. Th1 cells typically produce cytokines, such as IFN- $\gamma$ and IL-2, which are responsible for cell-mediated immune function protects cells against intracellular microbes. Th2 cells produce IL-4 and IL-6, which participate in atopic and allergic reactions [13]. T-bet and GATA-3 are two major Thspecific transcription factors that regulate expressions of Th1 or Th2 cytokine genes and play important roles in differentiation of Th cells [14]. Th1/Th2 imbalance plays a vital role in occurrence and development of AIT [15]. In this study, imbalance in Th1 and Th2 in EAT mice was improved by treatment with DHA. Therefore, DHA may partially alleviate AIT through modulation of Th1/Th2.

CXCL10 is a chemokine induced by IFN- $\gamma$, and it has been proposed as an inflammation marker of AIT [7-9]. CXCL10 may be a marker of a more powerful and more aggressive inflammatory response in the thyroid or cause destruction of thyroid tissues and hypothyroidism [16]. CXCL10 powerfully recruits lymphocytes expressing CXCR3 in inflamed tissues. Binding of CXCL10 chemokines and CXCR3 plays a vital role in Th immune response, which is responsible for organ-specific autoimmune diseases [7]. In this study, as observed through calcium flow experiments, DHA significantly inhibited interaction of CXCL10 and CXCR3.

NF-kB plays a vital role in inflammatory responses and immune reactions [17]. Inhibition of PI3K/AKT signaling pathway provides a new therapy for autoimmune diseases [18]; PI3K/AKT performs an important role in signal activation of NF-kB [18, 19]. Our results showed that DHA can inhibit transcriptional activation activity of NF-kB. Western blot results revealed that 
DHA inhibited expression of $\mathrm{p}-\mathrm{NF}-\mathrm{kB}$ by dramatically inhibiting phosphorylation of PI3K/AKT. Activation of CXCR3 increased activity of PI3K and its downstream pathway [20]. Based on the above experimental results, we hypothesize that DHA can inhibit CXCL10 binding to CXCR3, thereby inhibiting downstream signaling pathways of PI3K/AKT/NF-kB, which execute important roles in AIT.

\section{MATERIALS AND METHODS}

\section{Mice and experimental protocol}

Six-week-oldfemale C57BL/6J mice weighing $20 \pm 2 \mathrm{~g}$ were purchased from the Animal Center of the Academy of Military Medical Sciences (Beijing, China) and maintained in the Tianjin International Joint Academy of Biomedicine under specific pathogen-free conditions. All protocols conformed to Animal Ethics Committee of the Tianjin International Joint Academy of Biotechnology and Medicine.

\section{Chemicals and reagents}

DHA and sodium iodide were purchased from J\&K Scientific Ltd (Beijing, China). Porcine Tg, LPS, con A, complete Freund's adjuvant (CFA), and incomplete Freund's (IFA) adjuvant were provided by Sigma-Aldrich Co. Ltd (St. Louis, MO, USA). PMA was purchased from Beyotime Institute of Biotechnology (Jiangsu, China). IP-10 (CXCL10) was obtained from PeproTech EC Ltd. (Rocky Hill, NJ). CXCR3 plasmid was purchased from GeneCopoeia Inc (Rockville, MD, USA). FLIPR Calcium 6 Assay Kit was provided by Molecular Devices, LLC (Sunnyvale, CA). PI3K antibody, p-PI3K antibody, AKT antibody, p-AKT antibody, NF-kB/p65 antibody, p-NFkB/p65 antibody, and CXCR3 antibody were purchased from Affinity Bioreagents Co. Ltd (Colorado, USA). Secondary antibodies were purchased from EarthOx (San Francisco, USA).

\section{In vivo study}

Female C57BL/6J mice were immunized using porcine $\mathrm{Tg}$ as immunogen with Freund's as adjuvant. Porcine $\mathrm{Tg}(2 \mathrm{mg} / \mathrm{ml})$ was dissolved in phosphatebuffered saline (PBS) and emulsified 1:1 in CFA. Mice were immunized by subcutaneous injection of $\mathrm{Tg}(0.1 \mathrm{ml}$, s.c.) after emulsification on day 0 . A second subcutaneous injection was administered on day 14 using the same amount of Tg in IFA and once every two weeks for a total of six weeks. The blank group received subcutaneous injection of similar amounts of PBS and CFA or IFA at the same days $[21,22]$.

After immunization, C57BL/6J mice were randomly divided into six groups ( $n=6$ each): blank group (physiological saline; $n=6$ ), model group (physiological saline; $n=6)$, Dex group (4.5 $\mathrm{mg} / \mathrm{kg}$ of dexamethasone; $n=6)$, DHA (H) group (20 mg/kg of DHA; $n=6)$, DHA (M) group (10 mg/kg of DHA; $n=6)$ DHA (L) group (5 $\mathrm{mg} / \mathrm{kg}$ of DHA; $n=6)$. Dexamethasone and DHA were administrated by oral gavage once a day for 28 days to the treatment groups. The blank and model groups received the same volumes of physiological saline. All mice were weighed once every two days and sacrificed after four weeks of treatment.

\section{Spleen and thymus coefficients of mice}

Spleens and thymuses were removed from mice and weighed to obtain coefficients of spleen and thymus. Spleen index $(\mathrm{mg} / \mathrm{g})=$ spleen weight/body weight, and thymus index $(\mathrm{mg} / \mathrm{g})=$ thymus weight/body weight.

\section{Thyroid histology}

Thyroid tissues were removed and were fixed in $10 \%$ formalin in PBS for at least $24 \mathrm{~h}$ and stained by H\&E. Grading was performed blindly to the experimental groups from which tissues originated. Thyroids that exhibited inflammatory cell infiltration were considered cases of thyroiditis and subsequently subjected to H\&E staining, whereas the presence of mononuclear cell infiltration was scored as follows: 1) interstitial accumulation of inflammatory cells distributed between two or more follicles; 2) one or two foci of inflammatory cells reaching at least the size of one follicle; 3) $10 \%$ to $40 \%$ of the thyroid replaced by inflammatory cells; 4) $>40 \%$ of the thyroid replaced by inflammatory cells. Mean grades of EAT were assigned as follows: 0 to 1 , negative; 1 to 2 , mild; 2 to 3, severe; and 3 to 4 , acute [23].

\section{ELISA}

Blood samples were harvested from the orbit of each mouse at day 28 post-treatment to measure contents of serum IFN- $\gamma$, IL-2, IL-4, IL-6, TPOAb, and TGAb by using ELISA Kit (Mlbio, Shanghai, China). All tests were conducted according to manufacturer's instructions.

\section{Assay of lymphocyte proliferation}

Mice were sacrificed, and the spleen was aseptically separated. Spleens were separated into individual cells and filtered with a $40 \mu \mathrm{m}$-pore size cell strainer. Red blood cells were removed from cell suspensions by incubation for $5 \mathrm{~min}$ at room temperature in red cell lysis buffer and subsequently washed twice with PBS. Splenocytes were diluted in RPMI 1640 medium with $10 \%$ newborn bovine serum to a final concentration of $5 \times 10^{6}$ cells $/ \mathrm{mL}$. Spleen cells added with LPS $20 \mu \mathrm{g} / \mathrm{mL}$ or con A $20 \mu \mathrm{g} / \mathrm{mL}$ were seeded into a 96 -well plate and incubated at $37{ }^{\circ} \mathrm{C}$ and 
$5 \% \mathrm{CO}_{2}$. After overnight incubation, cells were treated with various concentrations of DHA. After incubation for $72 \mathrm{~h}$, cell viability was measured after addition of 25 $\mu \mathrm{L}$ 3-(4,5-dimethythiazol-2-yl)-2,5-diphenyltetrazolium bromide(MTT) at $37{ }^{\circ} \mathrm{C}$ for $4 \mathrm{~h}$, and then $150 \mu \mathrm{L}$ dimethylsulfoxide was added to dissolve formazan crystals [24]. Absorbance of each well was measured at $570 \mathrm{~nm}$ using a microplate reader (Multiskan ${ }^{\mathrm{TM}} \mathrm{FC}$, Thermo Fisher Scientifi, Waltham, MA, USA).

\section{Reverse transcription polymerase chain reaction (RT-PCR)}

Fresh spleens were removed from mice, rapidly frozen in liquid nitrogen, and then stored at $-80{ }^{\circ} \mathrm{C}$ prior to experiments. TRNzol Universal Reagent (TIANGEN BIOTECH, Beijing, China) was used to extract total RNA and for reverse transcription into cDNA after quantification by Nanodrop 2000 (Thermo Fisher Scientific, Wilmington, DE, USA). Primers were synthesized by AuGCT (Table 1). Quantitative PCR (Q-PCR) was performed using the Applied Biosystems 7900 Fast Real-Time PCR system (Applied Biosystems, Foster City, California, USA). Results were calculated using $\triangle \mathrm{CT}$ method, and ratios of all target genes in each group were based on their relation expression versus the level of GAPDH gene expression [25].

\section{Transient transfection and dual-luciferase assay}

Human embryonal kidney (HEK)-293 cells were obtained from KeyGen Biotech (Nanjing, China) and grown in DMEM with $10 \%$ fetal bovine serum at 37 ${ }^{\circ} \mathrm{C}$ in $5 \% \mathrm{CO}_{2}$. Cells were plated in 96-well plates at a concentration of $2 \times 10^{4}$ cells/well, and Lipofectamine 2000 transfection reagent was used for transfection. Recombinant plasmids NF-kB and TK were cotransfected into HEK-293 cells for $6 \mathrm{~h}$. Cells were cultured with various concentrations of DHA and/or $30 \mathrm{nM}$ PMA for $24 \mathrm{~h}$, and relative luciferase activity in cell extract was determined. Firefly luciferase activity was measured using ONE-Glo ${ }^{\mathrm{TM}}$ Luciferase Assay System (Promega Corp. Madison, WI) according to manufacturer's protocol [26].

\section{Intracellular $\mathrm{Ca}^{2+}$ concentration measurements}

HEK-293 cells stably transfected with CXCR3 were seeded into blank-walled 384-well plates. Cells were incubated for $12 \mathrm{~h}$ to allow plate seeding. Calcium 6 kit was used for dye loading. After cell attachment, culture medium was replaced with another culture medium containing different concentrations of DHA incubated with dye for $2 \mathrm{~h}$. A multifunctional microplate detecting instrument (Tecan Spark ${ }^{\mathrm{TM}} 10 \mathrm{M}$ ) was used to add CXCL10 chemokines $(100 \mathrm{ng} / \mathrm{ml})$ and to evaluate changes in florescence. $\mathrm{Ca}^{2+}$ concentrations were calculated after determining maximum and minimum ratios of fluorescence in the presence and absence of saturation levels of calcium [27].

\section{Chemotaxis assay}

Lymphocyte migration was evaluated by chemotaxis assays through a $5 \mu \mathrm{m}$ pore polycarbonate filter in 24well transwell chambers (Corning Costar, Cambridge MA). Lymphocytes were separated from spleens. Cell suspensions with various concentrations of DHA were placed in the upper chambers, whereas CXCL10 chemokines were added to the bottom chamber of the transwell $(0.6 \mathrm{~mL})$. The plate was incubated at $37{ }^{\circ} \mathrm{C}$ in $5 \% \mathrm{CO}_{2}$ atmosphere for $1 \mathrm{~h}$. Results are shown as chemotactic index which was calculated by dividing the percentage of cells migrated in the presence of CXCL10 by the percentage of cells migrated in its absence [27, 28].

\section{Western blot}

Lymphocytes induced by PMA (30 nM) were treated with 0,5 , and $10 \mu \mathrm{M}$ DHA for $48 \mathrm{~h}$. Proteins of harvested cells were extracted using RIPA buffer (Beyotime, Jiangsu, China). Proteins were separated by electrophoresis and transferred onto polyvinylidene difluoride membranes (Millipore, Bedford, MA, USA). Membranes were blocked and incubated with primary antibodies (PI3K, p-PI3K, AKT, p-AKT, NF-kB/p65, and $\mathrm{p}-\mathrm{NF}-\mathrm{kB} / \mathrm{p} 65)$. GAPDH was used as loading control. After incubation with respective alkaline phosphataseconjugated secondary antibodies (Santa Cruz, CA, USA), proteins were detected by an enhanced chemiluminescence Western blot kit (Amersham Corp., Buckinghamshire, United Kingdom) and exposed to a chemiluminescent film. Relative band intensity was quantified using Image J software (U.S. National Institutes of Health, Bethesda, MD, USA).

\section{Microarray}

Lymphocytes induced by PMA (30 nM) were untreated or treated with DHA for $24 \mathrm{~h}$. Cells were then washed thrice with PBS and treated with TRNzol Universal Reagent. Samples were sent to GENERGY Biotechnology (Shanghai) Co., Ltd. for expression microarray detection. GO analysis was used to detect DHA-induced changes in molecular functions, biological processes, and cellular components. STRING database was used to analyze protein-protein interactions, and KEGG analysis revealed inhibition of various signaling pathways.

\section{Statistical analyses}

All data were expressed as means \pm standard deviation (SD). Comparisons between groups were performed by one-way analysis of variance followed by 
Bonferroni post hoc test (SPSS software package version 17.0, SPSS Inc., Chicago, IL, USA). Level of significance was set at $\mathrm{P}<0.05$.

\section{CONFLICTS OF INTEREST}

The authors declare no conflicts of interest.

\section{FUNDING}

This study was founded by Foundation for the Author of National Excellent Doctoral Dissertation of China (Grant No. 201482), Tianjin science and technology innovation system and the condition of platform construction plan (Grant No.14TXSYJC00572), National Biomedical Special Project of International Innovation Park (Grant No.13ZCZDSY02600 and 13ZCZDSY03300),Tianjin Natural Science and Technology Fund (Grant No. 15JCYBJC26400), National Natural Science Funds of China (Grant No. 81572838, 81402973, and 81703581), Tianjin Science and Technology Project (Grant No. 15PTGCCX00140), Tianjin Science and Technology Project (Grant No.10ZCKFSY07200, 10ZCKFSY08800,13ZCZDS Y03800,13ZXCXSY1350 0 and 13ZCZDSY03300), Innovation fund for technology based firms (Grant No. 12ZXCXSY06500, 12ZXCXSY07200), Tianjin Research Program of Application Foundation and Advanced Technology (Grant No.13JCYBJC39600), and National Science and Technology Major Project (Grant No. 2017ZX09306007).

\section{REFERENCES}

1. Tomer Y, Huber A. The etiology of autoimmune thyroid disease: a story of genes and environment. J Autoimmu. 2009; 32:231-239.

2. Staii A, Mirocha S, Todorova-Koteva K, Glinberg S, Jaume JC. Hashimoto thyroiditis is more frequent than expected when diagnosed by cytology which uncovers apre-clinical state. Thyroid Res. 2010; 3:11.

3. Inada M, Nishikawa M, Naito K, Ishii H, Tanaka K, Imura H. Reversible changes of the histological abnormalities of the thyroid in patients with painlessthyroiditis. J Clin Endocrinol Metab. 1981; 52:431-435.

4. Caturegli P, De Remigis A, Rose NR. Hashimoto thyroiditis: clinical and diagnostic criteria. Autoimmun Rev. 2014; 13:391-397.

5. Vanderpump MP, Tunbridge WM, French JM, Appleton D, Bates D, Clark F, Grimley Evans J, Hasan DM, Rodgers H, Tunbridge F, Young ET. The incidence of thyroid disorders in the community: a twenty-year follow-up of the Whickham Survey. Clin Endocrinol.1995; 43:55-68.

6. Gul K, Dirikoc A, Kiyak G, Ersoy PE, Ugras NS, Ersoy $\mathrm{R}$, Cakir B. The association between thyroid carcinoma and Hashimoto's thyroiditis: the ultrasonographic and histopathologic characteristics of malignant nodules. Thyroid. 2010; 20:873.

7. Antonelli A, Ferrari SM, Giuggioli D, Ferrannini E, Ferri C, Fallahi P. Chemokine (C-X-C motif) ligand (CXCL)10 in autoimmune diseases. Autoimmun Rev. 2014; 13:272-280.

8. Lasagni L, Francalanci M, Annunziato F, Lazzeri E, Giannini S, Cosmi L, Sagrinati C, Mazzinghi B, Orlando C, Maggi E. An alternatively spliced variant of CXCR3 mediates the inhibition of endothelial cell growth induced by IP-10, Mig, and I-TAC, and acts as functional receptor for platelet factor 4. J Exp Med. 2003; 197:1537.

9. Antonelli A, Rotondi M, Fallahi P, Romagnani P, Ferrari SM, Buonamano A, Ferrannini E, Serio M. High levels of circulating CXC chemokine ligand 10 are associated with chronic autoimmune thyroiditis and hypothyroidism. J Clin Endocrinol Metab. 2004; 89:5496.

10. Yang L, Zhang D, Center A. Summary of dihydroartemisinin and its application for the treatment of lupus erythematosus. Chin Sci Bull. 2017.

11. Okayasu I, Kong YM, Rose NR. Effect of castration and sex hormones on experimental autoimmune thyroiditis. Clin Immunol Immunopathol. 1981; 20:240-245.

12. Wang YK, He HL, Wang GF, Wu H, Zhou BC, Chen XL, Zhang YZ. Oyster (Crassostrea gigas)hydrolysates produced on a plant scale have antitumor activity and immunostimulating effects in BALB/c mice. Mar Drugs. 2010; 8:255.

13. Neurath MF, Finotto S, Glimcher LH. The role of Th1/ Th2 polarization in mucosal immunity. Nat Med. 2002; 8:567-573.

14. Kiwamoto T, Ishii Y, Morishima Y, Yoh K, Maeda A, Ishizaki K, Iizuka T, Hegab AE, Matsuno Y, Homma S. Transcription factors T-bet and GATA-3 regulate development of airway remodeling. Am J Respir Crit Care Med. 2006; 174:142-151.

15. Elenkov IJ. Glucocorticoids and the Th1/Th2 Balance. Ann N Y Acad Sci. 2004; 1024:138.

16. Antonelli A, Fallahi P, Rotondi M, Ferrari SM, Romagnani P, Grosso M, Ferrannini E, Serio M. Increased serum CXCL10 in Graves' disease or autoimmune thyroiditis is not associated with hyper- or hypothyroidism per se, but is specifically sustained by the autoimmune, inflammatory process. Eur J Endocrinol. 2006; 154:651-658.

17. Strickland I, Ghosh S. Use of cell permeable NBD peptides for suppression of inflammation. Ann Rheum Dis. 2006; 65:iii75.

18. Foster JG, Blunt MD, Carter E, Ward SG. Inhibition of PI3K signaling spurs new therapeutic opportunities in inflammatory/autoimmune diseases and hematological malignancies. Pharmacol Rev. 2012; 64:1027.

19. Serasanambati M, Chilakapati SR. Function of nuclear factor kappa B (NF-kB) in human diseases-a review. South Indian J Biol Sci. 2016; 2:368. 
20. Bonacchi A, Romagnani P, Romanelli RG, Efsen E, Annunziato F, Lasagni L, Francalanci M, Serio M, Laffi G, Pinzani M. Signal transduction by the chemokine receptor CXCR3: activation of Ras/ERK, Src, and phosphatidylinositol 3-kinase/Akt controls cell migration and proliferation in human vascular pericytes. J Biol Chem. 2001; 276:9945-9954.

21. Nan X, Gang C, Min L, Ye X, Pan Y, Ge J, Mao Y, Wang H, Jian W, Xie S. Anti-inflammatory effects of luteolin on experimental autoimmune thyroiditis in mice. Exp Ther Med. 2016; 12:4049.

22. Cui SL, Yu J, Shoujun L. Iodine intake increases IP-10 expression in the serum and thyroids of rats with experimental autoimmune thyroiditis. Int $\mathrm{J}$ Endocrinol. 2014; 2014:581069.

23. Tang H, Mignon-Godefroy K, Meroni PL, Garotta G, Charreire J, Nicoletti F. The effects of a monoclonal antibody to interferon- $\gamma$ on experimental autoimmune thyroiditis (EAT): prevention of disease and decrease of EAT-specific T cells. Eur J Immunol. 1993; 23:275-278.

24. Frew IJ, Hammond VE, Dickins RA, Quinn JM, Walkley CR, Sims NA, Schnall R, Della NG, Holloway AJ, Digby
MR. Generation and analysis of Siah2 mutant mice. Mol Cell Biol. 2003; 23:9150-9161.

25. Jin X, Wang S, Zhao X, Qian J, Fan C, Jing L, Shan Z, Teng W. Coumestrol inhibits autoantibody production through modulating Th1 response in experimental autoimmune thyroiditis. Oncotarget. 2016; 7:52797-52809. https://doi. org/10.18632/oncotarget.10353.

26. Hwang YP, Yun HJ, Kim HG, Han EH, Lee GW, Jeong HG. Suppression of PMA-induced tumor cell invasion by dihydroartemisinin via inhibition of PKCalpha/Raf/MAPKs and NF-kappaB/AP-1-dependent mechanisms. Biochem Pharmacol. 2010; 79:1714.

27. Sebastiani S, Allavena P, Albanesi C, Nasorri F, Bianchi G, Traidl C, Sozzani S, Girolomoni G, Cavani A. Chemokine receptor expression and function in CD4+ T lymphocytes with regulatory activity. J Immunol. 2001; 166(2):996

28. Juarez J, Dela PA, Baraz R, Hewson J, Khoo M, Cisterne A, Fricker S, Fujii N, Bradstock KF, Bendall LJ. CXCR4 antagonists mobilize childhood acute lymphoblastic leukemia cells into the peripheral blood and inhibit engraftment. Leukemia. 2007; 21:1249-1257. 